\title{
Impacto de la normalización de la política monetaria de la FED en el acceso al crédito de las empresas latinoamericanas
}

\author{
Impact of the normalization of monetary \\ policy on the access FED to credit of Latin \\ American firms
}

DOI: https://doi.org/10.17981/econcuc.42.1.2021.Econ.3

Artículo de investigación.

Fecha de recepción: 21/04/2020

Fecha de devolución: 25/09/2020

Fecha de aceptación: 05/10/2020

Fecha de publicación: 12/10/2020

\section{Héctor Alberto Botello Peñaloza \\ Universidad Industrial de Santander. Bogotá, D.C. (Colombia) hectoralbertobotello@gmail.com}

\section{Resumen}

El financiamiento de las empresas (interno o externo) es lo que potencia el tejido productivo de los países, así entonces esta investigación tiene como objetivo estimar el impacto de la normalización de la política monetaria de la Reserva Federal de Estados Unidos sobre el acceso al crédito de las empresas latinoamericanas. Se implementa un modelo de elección discreta sobre un panel longitudinal para 25 mil firmas en 12 países entre el 2010 y 2017. Se introducen diversas variables internas y externas a las firmas. Adicionalmente, se incluyen variables sobre la estructura bancaria de los países, la variación de las tasas de interés nacionales y de las FED. Entre los resultados se obtiene que los determinantes más relevantes para acceder al crédito son las características internas de las firmas. Por otro lado, el cambio de la política monetaria de la Reserva federal no fue significativa en las estimaciones. Adicionalmente se encontró que mayores niveles de competencia en el sector financiero disminuyen la cantidad de fondos internos utilizados por las empresas para financiar la actividad productiva de la firma un $12 \%$.

Palabras clave: Financiamiento externo; Finanzas Corporativas; Regulación; Competencia financiera

\begin{abstract}
The financing of companies (internal or external) is what enhances the productive fabric of the countries, so then, this research aims to estimate the impact of the normalization of the monetary policy of the United States Federal Reserve on the access to credit of Latin American companies. A discrete choice model is implemented on a longitudinal panel for 25 thousand firms in 12 countries between 2010 and 2017. Various internal and external variables are introduced for the firms. Additionally, variables on the banking structure of the countries, the variation of national interest rates and the FED are included. The results show that the most relevant determinants of access to credit are the internal characteristics of the firms. On the other hand, the change in the monetary policy of the Federal Reserve was not significant in the estimates. Additionally, it was found that higher levels of competition in the financial sector reduce the amount of internal funds used by companies to finance the firm's productive activity by $12 \%$.

Keywords: External financing; Corporate finance; regulation;
\end{abstract} financial competition 


\section{INTRODUCCIÓN}

El financiamiento de las empresas permite la expansión del tejido productivo de los países al permitir la generación y mantenimiento de nuevas cadenas actividades económicas (Greenwood, Sánchez \& Wang 2013). Las fuentes de estos recursos se pueden obtener mediante recursos internos o externos. El autofinanciamiento de la firma puede originarse desde los accionistas de las empresas, los excedentes del flujo de caja o de las reservas acumuladas por las utilidades de años anteriores.

Desde otro punto de vista, el financiamiento externo puede obtenerse a través de a través de créditos bancarios, bonos, emisiones accionarias entre otros. La evidencia enseña que el crecimiento de las empresas por medio de los fondos externos es más dinámico frente al causado mediante los internos, pues no se debe esperar el periodo de acumulación mediante los fondos prestables provenientes de los socios. Igualmente, el endeudamiento externo puede traer beneficios fiscales al disminuir el impacto tributario (Lago, López \& Saurina, 2007; Ramírez \& Sarmiento, 2020; Urdaneta, Pérez \& Acurero, 2018). En consecuencia, el acceso a fuentes de financiamiento externas ha sido asociado a mayores niveles de crecimiento y desarrollo económico, como se presenta en la Figura 1 (Rice \& Strahan, 2010).

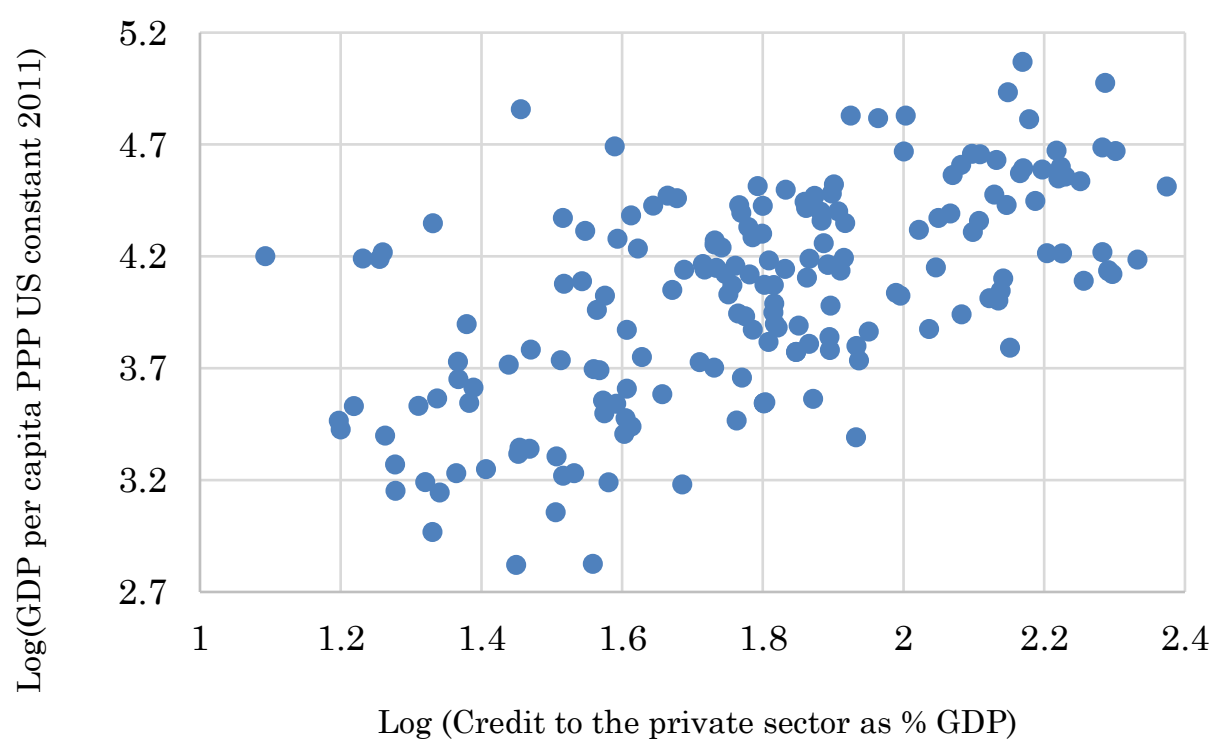

Figura 1. Profundización financiera y desarrollo económico, 2015.

Fuente: Elaboración del autor con datos del FMI y el Banco Mundial (BM, 2020).

Sin embargo, existen fallas de mercado que afectan el acceso de las compañías a los fondos prestables de terceros. En este sentido, según Jiménez y Manuelito (2011) la región latinoamericana enseña un nivel de profundización financiera menor que sus pares. Esta problemática es evidencia de los altos costos de financiamiento lo cual impone barreras al crecimiento vía una estructura empresarial centrada en el uso de fondos internos. Igualmente, el cierre de las fuentes formales de crédito empuja a las PYMEs a solicitar recursos en los mercados informales donde los tipos de interés son elevados y los plazos de maduración cortos (Quicazan, Fernández y Estrada, 2012). Lo anterior, restringe el crecimiento de la firma y conlleva a círculos de informalidad con baja productividad (De Haas \& Van Horen, 2009). 
Igualmente, dentro del plano macroeconómico, la política monetaria es una de las principales herramientas que tienen los Bancos Centrales para lograr una mayor dinamización del crédito mediante la dinámica de las tasas de interés y el encaje bancario. Sin embargo, las decisiones de política tienen efectos tanto domésticos como externos, reflejados en las variaciones de los tipos de cambio y los flujos de capital. Estos impactos serán mayores si las decisiones son tomadas por las grandes economías, dada su importancia en los portafolios internacionales y la dependencia de otras economías en proceso de crecimiento (Ramírez \& Sarmiento, 2020).

Por lo anterior, los factores mencionados han sido tema de interés para los investigadores. Sin embargo, la tarea de analizar estas variables es compleja dado que son resultados de la interacción entre las características internas y los contextos externos a las firmas (Botello, 2015). Por ejemplo, estas pueden provenir por la imposibilidad de las firmas de cumplir los estándares para la devolución del capital, la concentración financiera, altos tipos de interés o la falta de información que permita a las instituciones financieras valorar los riesgos de las empresas (Vives, 2001; Maudos, 2016).

En este sentido el presente documento desea contribuir a la literatura investigando el impacto de la normalización de la política monetaria de EUA sobre la concentración bancaria y el acceso al crédito de las empresas latinoamericanas. En pro de lo anterior, se estiman modelos probabilísticos con base en un panel longitudinal de 25 mil firmas en 12 países de Latinoamérica.

La metodología consigue tener en cuenta un rango amplio de características de las empresas con el fin de estimar su impacto sobre la probabilidad de acceso a un crédito. Las variables puestas en el modelo se basan en la literatura existente sobre la temática de determinantes del financiamiento externo de las empresas (Granda, 2012; Gámez, Morales \& Ramírez, 2018). En el caso particular de esta investigación se utilizan características de las compañías, así como factores macroeconómicos, la competencia bancaria de cada país y el cambio de las tasas de interés externas de la reserva federal.

Las estimaciones evidencian que el volumen de ventas (tamaño) y la capacidad tecnológica de la misma son las variables más relevantes para el acceso a los mercados de financiamiento debido a su habilidad para poseer ventajas competitivas dentro de las unidades productivas, permitiéndoles generar flujos de caja sostenibles. Igualmente, los resultados apuntan a que los países con una menor cantidad de crédito disponible y alta concentración bancaria están asociadas con la disminución en la probabilidad del acceso al financiamiento. Estos resultados van en línea con los alcanzados por otros trabajos en varios países (Bikker, Van Rixtel y Kok-Sørensen, 2008).

Esta investigación se encuentra dentro de un conjunto de investigaciones relacionadas con la inclusión financiera, el acceso al crédito y la competencia dentro del sector financiero. Carbó-Valverde, Rodríguez-Fernández y Udell (2009) calculan el índice de concentración de Lerner para examinar la relación entre la competencia bancaria y el acceso a la financiación en España. Beck, Degryse y Kneer (2004) utilizan datos de 73 países para un período y descubren que la alta concentración tiene un impacto negativo en el acceso de las empresas. Resultados que se han alcanzado igualmente en otros países (Martínez, Zuleta, Misas y Jaramillo, 2016; De la Torre, 2018). 
Ryan, O’Toole y McCann (2014) examinan el poder de mercado y las restricciones crediticias de 118.000 PYMEs en 20 países europeos durante el período 2005-2008. Encuentran un incremento del poder de mercado traducido en mayores restricciones financieras. Love y Martínez (2014) estudian encuestas de varios años a nivel de la firma de 53 países buscando estimar el impacto de la falta de competencia en el acceso a la financiación. Encuentran que la baja competencia medida por los altos valores del índice Lerner o del indicador Boone, disminuye el acceso de las empresas a la financiación.

Con vista de lo anterior, este trabajo aporta a la literatura de varias maneras. En primer lugar, examina el vínculo relacional entre la competencia bancaria y el acceso a la financiación, utilizando datos de paneles longitudinales a nivel de la firma dentro del entorno latinoamericano considerando las afectaciones provenientes de la influencia de la política monetaria de la Reserva Federal de EUA. El panel permite controlar las características que podrían influir en el acceso de las unidades productivas a la financiación y comprobar si las relaciones entre la competencia, el acceso a la financiación y la política monetaria están presentes a lo largo del tiempo y en consideración de cada país.

Esto es importante porque la calidad de las instituciones o las normas varían significativamente y pueden afectar tanto al acceso a la financiación como al grado de competencia. Estos indicadores cambian con el tiempo y son captados por los efectos fijos en las estimaciones. Los trabajos de Carbó-Valverde et al. (2009), Nguyen (2019), Claessens y Laeven (2005), González y González (2008) y De Miguel y Pindado (2001) son de un solo año o un país especifico.

En segundo lugar, este documento busca un vínculo relacional entre la competencia bancaria, el acceso a la financiación y los cambios en la política monetaria externa utilizando datos de paneles longitudinales a nivel de la firma, lo cual permite controlar las características dentro de cada firma que podrían influir en el acceso de las empresas a la financiación. El trabajo de Love y Martínez (2014), aunque amplio, no conserva la homogeneidad, por lo tanto, las variaciones ocurridas dentro de la muestra pueden afectar las inferencias sobre la probabilidad de acceso al crédito.

En tercer lugar, se introducen en los modelos indicadores objetivos del acceso de las firmas a la financiación en lugar de estimar indicadores sustitutivos, como en Claessens y Laeven (2005) y Beck et al. (2004). El indicador captura si una empresa posee productos de crediticios, como un préstamo, sobregiro o una línea de crédito. Por ejemplo, en Antoniou, Guney y Paudyal (2008) se aproximan indicadores de financiamiento directamente desde el balance, pero esta medida excluye a las otras firmas que logran acceder al mercado financiero y no están reflejado en las normas contables.

El documento está organizado de la siguiente manera, primero, se expone una breve situación del financiamiento empresarial en América Latina. En la tercera sección se presenta la evolución de la política monetaria en EUA y su posible impacto en Latinoamérica. A continuación, se presentan los datos y el resumen estadístico de la encuesta utilizada, seguido por la presentación del modelo de data panel longitudinal. En la cuarta sección se presentan los resultados de las estimaciones. Finalmente, en las conclusiones se discuten las implicaciones de los hallazgos. 


\section{CONTEXTo TEÓRICO}

\section{El financiamiento empresarial en América Latina}

El acceso al crédito en la región es limitado y presenta América Latina es una región rezagada en términos de profundización financiera (Figura 2). En América Latina el crédito corresponde al 48\% del PIB (Chortareas, Garza-García, y Girardone 2010). En otras regiones desarrolladas se encuentra por encima del $100 \%$ del PIB. Entre los países de la región, Chile y Panamá cuentan con alrededor del 78\% y $92 \%$ del PIB.

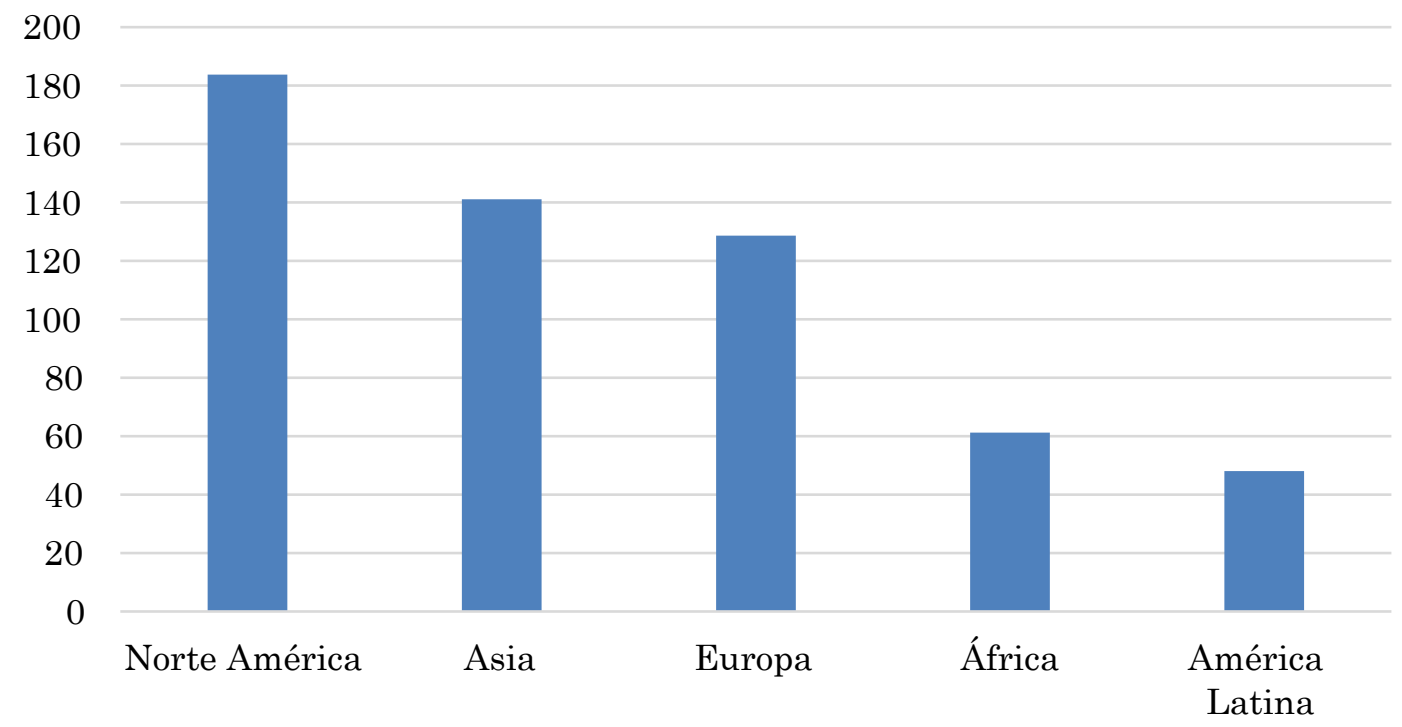

Figura 2. Crédito al sector privado como porcentaje del PIB, 2015.

Fuente: Elaboración del autor con datos del FMI y el Banco Mundial (BM, 2020).

Algunos autores han atribuido el anterior problema a la concentración en pequeñas empresas y la informalidad de las economías. Según Arazi y Baralla (2012) estiman que las Pequeñas y Medianas Empresas (PYMEs) aportan cerca del 67\% del empleo y $40 \%$ del valor agregado. Sin embargo, $40 \%$ de estas unidades productivas son informales ya que sus trabajadores no cotizan a pensión o tienen contabilidad y no pagan impuestos (Cárdenas y Mejía, 2007).

Acorde a Jiménez y Manuelito (2011) las restricciones del financiamiento pueden estar basado en el bajo número de productos de crédito y el alto riesgo causado por la baja productividad de las firmas. En este sentido, el escaso porcentaje de empresas que logran acceder al financiamiento externo se ve afectado significativamente por cualquier cambio regulatorio o de la estructura financiera de los países en los que se encuentren.

Por lo tanto, la presente investigación parte de un contexto latinoamericano donde el crédito a las empresas se encuentra limitado y donde su estructura financiera se verá afectada por políticas monetarias del exterior. El artículo propone contribuir al estudio de este tema utilizando la siguiente metodología. 


\section{Impacto de la política monetaria estadounidense en los países de Latinoamérica}

En el plano de la economía internacional, es visible que las decisiones adoptadas por cada uno de los países tienen repercusiones en otras economías, como por ejemplo las decisiones de política económica de los Estados Unidos, dado que representa la mayor economía mundial, fuente de fondos comerciales y de inversión, a la vez que posee la moneda internacional de intercambio, el dólar.

Durante los últimos dos siglos, Latinoamérica ha tratado de mantener relaciones comerciales, diplomáticas, sociales, etc., con Estados Unidos por causa de los grandes beneficios que atañe en pro del crecimiento, el desarrollo y el bienestar de las naciones. Sin embargo, así como las economías se pueden beneficiar de esta relación, también se verán perjudicada en caso tal que uno de los países presente coyunturas negativas significativas que afecten rubros específicos de la economía.

En este orden de ideas, las políticas económicas de Estados Unidos han probado ser fuente de influencia en los mercados y las políticas económicas de otros países (Eichengreen \& Gupta, 2013; Aizenman, Binici \& Hutchison, 2014; Andritzky, Bannisterb \& Tamirisab, 2007; Ramírez \& Sarmiento, 2020). Ejemplo los bonos de deuda público están expuestos a las variaciones de las tasas de interés de Estados Unidos alterando el interés de los inversionistas por estos y los del tesoro estadunidense. Sin embargo, en la última década, los gobiernos se han puesto a cubierto de esta tendencia emitiendo una mayor proporción de deuda pública denominada en moneda local (Miyajima, Mohanty \& Chan, 2015; Arora \& Cerisola, 2001).

En otro sentido, la política monetaria también ha sido señalada de ser fuente de distorsión en los precios de los activos y los tipos de cambios (Bernanke \& Kuttner, 2005); de esta manera, es necesario entender la manera en que funciona la política monetaria estadounidense para posteriormente entender los efectos de las medidas que adoptan. La política monetaria estadounidense es manejada por la Reserva Federal (FED), la cual cuenta con las tasas de descuento y las operaciones de mercado abierto. Con estos instrumentos la FED busca mantener el control y el equilibrio de las reservas, la oferta monetaria y los tipos de interés, que van a determinar por consiguiente el crecimiento económico.

El principal impacto que tienen las economías latinoamericanas ante las decisiones de política monetaria estadounidense, son el tipo de cambio, los flujos de capital y los posteriores choques directos en la balanza comercial. En este sentido, Eichengreen y Gupta (2013) y Rai y Suchanek (2014) estudiaron el impacto de los retiros de los estímulos monetarios declarados en mayo de 2013 por la FED, sobre los tipos de cambio, reservas internacionales y precios de los activos. Los autores encuentran que después del anuncio apareció una menor liquidez en los mercados financieros e incrementos en los déficits comerciales.

El artículo de Aizenman et al. (2014) emplean un marco de panel usando datos diarios, encontrando que los precios de los activos de los mercados emergentes respondieron más a las declaraciones del presidente de la FED sobre el retiro de los estímulos, así como que los países con fundamentos más fuertes, tenían activos que respondían más a las noticias que los países con niveles menores de reservas y flujos de capital. 
Las perturbaciones de los anteriores activos afectan los objetivos de política monetaria en otros países, por esto es preocupación de los bancos centrales, las brechas establecidas entre sus tasas de interés frente a las sus tasas y las estadunidenses, dado que estas causan movimientos de capital desestabilizadores y tipos de cambio volátiles. Lo anterior influye las sendas de crecimiento e inflación de los países. Desde el punto de vista que le interesa a esta investigación, pocos trabajos han abordado el efecto de la política monetaria estadounidense sobre las decisiones de los bancos centrales enfocadas a los mercados emergentes y en Latinoamérica.

En un trabajo para veinte economías emergentes, Avdjiev y Takáts (2014) encuentran que para 16 de 20 países, la tasa de los fondos federales es relevante y trasciende a las condiciones internas de los países. Los resultados de las estimaciones muestran que las tasas de interés consiguieron estar en línea a regla de Taylor en la última década, resultado igual al reportado por Hofmann y Bogdanova (2012). Finalmente resalta que el retiro de los estímulos contribuyó a reducir las diferencias que existían, pero aún se conserva una alta dispersión entre países.

En este orden de ideas, desde principios de siglo la reserva federal ha mostrado dos tendencias marcadas a causa de la crisis internacional de 2007: el relajamiento cuantitativo (QE) y el retiro de los estímulos monetarios (tapering). A continuación, se exponen brevemente sus consecuencias en los mercados financieros y su posterior influencia en las políticas monetarias de los países de América Latina.

\section{Relajamiento Cuantitativo}

El relajamiento cuantitativo, es un conjunto de acciones por parte de la FED que nacen como respuesta a la crisis económica del 2007-2008 que afectó a economías desarrolladas, en desarrollo y emergentes, con el objetivo de mejorar el crecimiento de la economía y los indicadores de desempleo, mediante el mantenimiento en bajos niveles de las tasas de interés de mediano y largo plazo, logrando aumentar la oferta de créditos. El relajamiento cuantitativo, nace como una reacción de las autoridades frente a la poca efectividad que venían presentando las políticas monetarias convencionales en la estimulación de la economía nacional y en los bajos niveles de endeudamiento buscados por los diferentes sectores (Fawley \& Neely, 2013).

El relajamiento cuantitativo, se desarrolló para el caso de los Estados Unidos en 3 rondas, con el objetivo de rescatar el sistema financiero nacional. La primera ronda o QE1 se implementó en noviembre del 2008, por parte del Comité Federal de Mercado Abierto de la Reserva Federal de EEUU, cuyo objetivo fue la expansión monetaria mediante la compra de deuda del gobierno de Estados Unidos, así mismo, la entidad opta por comprar valores respaldados por hipotecas (compra de deuda y activos financieros privados, conocidos en algunos casos como activos tóxicos o bonos basura) (García \& Baeza, 2016).

La segunda ronda del relajamiento cuantitativo (QE2) se ejecutó dos años después del QE1 en noviembre del 2010, para esta ronda la Reserva Federal decide seguir comprando deuda pública del gobierno por 600,000 millones de dólares. El QE2 a diferencia del QE1 duró menos de un año en ejecución, dándose por terminado en el mes de 
junio del 2011. Como respuesta a la finalización del QE2 la Reserva Federal lanza la Operación Twist, que buscaba incentivar la inversión y reducir las tasas de interés de largo plazo, por medio del canje de bonos de largo plazo por bonos de corto plazo por una cantidad de 400,000 millones de dólares, esta se mantuvo hasta finales del 2012.

La tercera ronda o QE3 inició en septiembre de 2012, acorde a la FED, esta etapa no tendría una fecha de fin. Sin embargo, el Banco Central iniciaría su merma hacia enero de 2014 y se esperaba su cierre total en octubre. Sus fondos iniciales eran de 40,000 millones de dólares mensuales. No obstante, estos se incrementaron a 85 mil millones de dólares cada mes. Como consecuencia de este incremento de la oferta monetaria en los Estados Unidos, la tasa de los bonos a diez años disminuyó desde el 3,5\% en 2011 a un rango entre 1,4\% y 1,7\% hacia el tercer trimestre de 2012.

Según Córdoba (2017), el mayor tipo de interés entre los países emergentes y EUA, llevó al incremento de los flujos de capital hacia los países emergentes las entradas de capital de portafolio con la posterior apreciación de las monedas regionales. Esta conllevo una caída de las tasas de interés de los activos financieros locales; estímulo a la dinámica del crédito, y alza en los precios de activos reales y financieros.

\section{Retiro de los estímulos (Tapering)}

Luego de ser implementado el programa de Relajamiento Cuantitativo y de evidenciarse una recuperación en la economía estadounidense, con la disminución en la tasa de desempleo del 7\% al 6,2\%, el aumento del gasto de los consumidores y una tasa sostenida de crecimiento en la economía americana de promedio el $2 \%$, la banca central estadounidense decide retirar los estímulos monetarios, dándose a conocer tras un anuncio realizado el 22 de mayo de 2013 con el fin de reducir los estímulos a la economía interna estadounidense, que en su momento, fueron de gran utilizad para lograr estabilizar la economía de los choques ocasionados por la crisis, mediante la inyección de liquidez mediante la adquisición de bonos públicos principalmente.

El tapering (disminución en los fondos de liquidez), fue el programa destinado a reducir en 10 mil millones de dólares al mes la compra de bonos públicos e hipotecarios, llegando a una generación de fondos de 75 mil millones de dólares a partir de enero de 2014. Estas disminuciones se fueron haciendo de forma paulatina, hasta terminar el programa en octubre del 2014 (García \& Baeza, 2016).

Después de dos años del inicio del tapering, la banca central estadounidense incrementa su tasa de fondos al rango del 0,25\% - 0,50\% en diciembre de 2015. Hacía nueve años que el banco central de Estados Unidos no subía el precio del dinero y siete en donde se mantenían tasas de casi a cero por ciento. A finales del 2016, la FED vuelve a sorprender con un nuevo aumento, ubicándose así la tasa de interés en 0,75\%. La decisión fue motivada al igual que el año anterior, porque los indicadores evidenciaban que el mercado laboral continúa fortaleciéndose y la actividad económica presentaba una continua expansión.

A partir del ajuste la economía mundial entró en una fase de volatilidad en los mercados financieros y en riesgo de crisis a los países emergentes (García \& Baeza, 2016). Para el caso de las economías latinoamericanas el alza en las tasas de inte- 
rés provocó una salida de la inversión extranjera re-direccionada hacia los Estados Unidos, presentando devaluaciones generalizadas en los tipos de cambios. A causa del componente de bienes importados en las canastas de compra de los países, pass through tipo de cambio se tradujo en un incremento de la inflación, un incremento de las tasas de interés, disminución del crecimiento en el crédito y reducciones en los precios de los activos.

Visto el canal de trasmisión de la política monetaria americana y sus acciones en años recientes, se investiga en este trabajo si el acceso al crédito de las empresas latinoamericanas fue afectado por el retiro de los estímulos y el incremento de las tasas de interés. El método de análisis se presenta a continuación.

\section{Metodología}

\section{Fuente de datos}

\section{- Datos bancarios}

Las superintendencias bancarias de cada uno de los países son la fuente de datos primaria tomadas para el análisis. La muestra cubre bancos comerciales, cooperativas de crédito y entidades hipotecarias contenidos en el periodo de 2010-2017. No se incluyen otras sociedades financieras como las agencias de inversión, las sociedades y agencias de valores, ni los organismos públicos especializadas. El monto de los activos financieros es tomado individualmente para el cálculo de los indicadores de concentración. Los tipos de interés fueron asociados a las tasas de intervención puestas por cada Banco Central de los países estudiados.

La información sobre el tamaño total de los activos se tomó a partir de los datos de la Federación Latinoamericana de Bancos. Los otros indicadores escogidos para caracterizar el sector financiero de los países son el margen de utilidad, el número de sociedades financieras, el crédito y los depósitos bancarios como porcentaje del PIB entre los años 2010-2017. Finalmente, la tasa de los fondos federales se toma del sistema de información de la reserva federal de San Luis.

\section{- Datos de las características de las empresas}

Los microdatos a nivel de la firma son provistos por el Banco Mundial (BM) a través de su Enterprises Survey (BM, 2020). Esta encuesta es de tipo estratificado, recogiendo datos de los gerentes de las empresas. Este documento utiliza la información de los años 2010 y 2017 de los países latinoamericanos: En total se contabilizan 25 mil firmas. (En el Anexo 1 se encuentra la tabla donde también expone la cantidad por tamaño de la empresa).

Los módulos de información abarcan desde variables internas y externas a la compañía. En general, el indicador de acceso captura si una empresa utiliza productos financieros, como un préstamo, sobregiro o una línea de crédito. Por su parte, la estructura de capital se caracteriza por las variables de porcentaje de los activos que se encuentra financiado por fuentes internas, bancos u otras fuentes (Rivera, 2007). 


\section{- Modelo}

En primer lugar, se realiza una descripción de los datos más relevantes encontrados en las fuentes de información señaladas sobre la estructura financiera y acceso al crédito de las empresas. A continuación, se pasa a presentar los modelos econométricos que servirán para validar las hipótesis buscadas por la investigación.

\section{- Midiendo el impacto de la normalización de la política monetaria de EUA en el crédito}

Para poder inferir un efecto relacional entre los movimientos en la política monetaria de EUA y el acceso al crédito se utiliza un modelo de variables dicotómicas dentro de un modelo de data panel de efectos fijos. Los modelos de data panel incrementan los grados de libertad y disminuyen el problema de colinealidad entre las variables explicativas, por lo tanto, mejora la eficiencia de las estimaciones econométricas (Boz, Menéndez, Orgaz y Prior, 2015).

El acceso al crédito se plantea como una variable dicotómica que toma valores de 1 si la empresa obtuvo fondos externos (bancos $u$ otras fuentes formales de financiamiento) y cero en caso contrario. Para esta estimación se utilizó un modelo de elección discreta. Para este trabajo se aplica la función de estimación de probabilidad del modelo lineal truncado. En este modelo se encuentra una variable no observable, $I$, que traza el comportamiento de la variable independiente $Y$. Esta se encuentra explicada por un vector de variables independientes $X_{i}$. La variable $Y$ presenta ciertos valores si la no observable cruza ciertos lumbrales (ecuación 1).

$$
Y_{i}=\left\{\begin{array}{lll}
1 & \text { si } I_{i}^{*}>0 & \text { lo que ocurre cuando } X_{i} \beta+\varepsilon_{i}>0 \\
0 & \text { si } I_{i} *>0 & \text { lo que ocurre cuando } X_{i} \beta+\varepsilon_{i}<0
\end{array}\right.
$$

Donde el supuesto sobre la distribución uniforme de $\mathcal{E}$, se implementa el Modelo Lineal de Probabilidad truncado ${ }^{1}$. Bajo este enfoque el modelo quedaría definido de la siguiente manera (ecuación 2):

$$
P_{i}=\operatorname{Pr} o b\left(Y_{i}=1\right)=\operatorname{Pr} o b\left(I_{i}^{*}>0\right)=\operatorname{Pr} o b\left(X_{i} \beta+\varepsilon_{i}>0\right)=\mathrm{F}\left(X_{i} \beta\right)
$$

La variable dependiente se expone como una probabilidad de ocurrencia del fenómeno analizado (Pérez, 2004). Para esta investigación la función del modelo probabilístico seria (ecuación 3):

$$
Y=f(S, X) \text { donde } Y_{i}=\alpha+\beta_{i} X_{i}+\varepsilon
$$

\footnotetext{
${ }_{1}$ Si se distribuye como una normal con media cero y varianza uno, el modelo generado será un Probit; mientras que, si se supone que se distribuye como una curva logística, se trataría de un modelo Logit (Medina, 2003).
} 
La variable dicotómica dependiente se categorizada como 1 cuando la empresa pudo acceder al financiamiento externo en el año $i$ y cero en caso contrario. Por su parte $X$ representa variables internas y externas a la firma que se tomaron de la literatura. Se resumen de la siguiente manera:

H1: Volumen de ventas y número de empleados.

H2: Años del establecimiento en funcionamiento.

H3: Capacitación de los trabajadores en cursos de calidad, posee email, tiene página web o certificaciones de calidad.

H4: Características de las organizaciones: Experiencia del gerente, concentración accionaria de la empresa, tenencia de cuenta de ahorros.

H5: Rama de actividad económica y tipo de ciudad donde se ubica la empresa.

H6: Indicadores de la estructura bancaria. El HHI del sector, Activos, margen de utilidad, el número de entidades financieras, el crédito y los depósitos bancarios como porcentajes del PIB.

H7: Tasa de los fondos federales de la FED de EUA en ambos periodos de tiempo.

\section{Modelo data panel}

Con base en esta metodología se arma un panel longitudinal con los datos del banco mundial. Un conjunto de información de este tipo incluye individuos (departamento, empresas, ciudades entre otros) dentro de una serie de períodos en el tiempo; es decir se combinan dos dimensiones: la temporal y estructural. En este trabajo, los elementos temporales serían los años desde del 2010 y 2017 y la estructural, las 25 mil empresas de las que se encuentra información.

La principal ventaja de aplicar y estudiar los datos en panel frente a un modelo de regresión lineal multivariado, es el de poder capturar la heterogeneidad no observable del fenómeno a estudiar, sea entre los individuos o el tiempo investigado (Arellano y Bover, 1990). Según Burdisso (1997), los efectos individuales específicos, son aquellos que afectan de manera particular a cada uno de los individuos, siendo invariables en el tiempo y que afectan de manera directa las decisiones que tomen dichos agentes. Usualmente se identifica este tipo de efectos con cuestiones de capacidad empresarial, eficiencia operativa, capitalización de la experiencia, acceso a la tecnología, etc. Por su parte, los efectos temporales son los que afectan a todos los casos de estudio de manera uniforme pero que varían constantes en el tiempo. Esta clase se puede relacionar a, por ejemplo, choques macroeconómicos o introducción de políticas que pueden afectar por igual a todos los departamentos.

Teniendo en cuenta supuestos del modelo y de los parámetros, se puede llegar a la forma de estimación (ecuación 4):

$$
Y_{i t}=\alpha_{i t}+\beta X_{i t}+u_{i t}
$$


Lo anterior, se representan con $i$, y las temporales con que van desde el 1994 al 2015. La variable dummy de acceso al credito se representa con $Y$ frente a el vector de variables internas y externa a las firmas. Los coefientes indican la magnitud de las relaciones de estas las variables está determinada por el $\beta$. Se espera rechazar la hipotesis nula de este coeficiente al menos al 5\% de significancia. Igualmente, y basado en la parte conceptual del trabajo, se espera que este sea negativo para las tasas de interés de la FED. Es decir que si aumentan las tasas de interés afectan el acceso al crédito al encarecerlo.

Asimismo, la lectura de los modelos de datos de panel se realiza usualmente a través de las hipótesis en el comportamiento en sus componentes de errores. El término de error incluido en la ecuación 1, puede descomponerse de la siguiente manera (ecuación 5):

$$
U_{i t}=\mu_{i}+\delta_{t}+\varepsilon_{i t}
$$

Donde representa los efectos especificos no observables que varian entre los individuos, pero no en el tiempo. Mientras que corresponde a los efectos temporales y al error puramente aleatorio. Es posible establecer diferentes casos del modelo, según las hipótesis que se tejan alrededor de. Si su valor es cero, no existe heterogeniedad no observable entre las firmas. El error se puede interpretar como el error dentro de un modelo de regresión lineal general, cumpliendo con todos los supuestos de este. La segunda alternativa consiste en suponer a es diferente a cero y es fijo para todas las empresas, es decir, la heterogeneidad no observable se incorporará a la constante del modelo.

La tercera forma es tratar a como una variable aleatoria no observable que varía entre individuos, pero no en el tiempo; en este caso tendremos que cada empresa posee características diferenciadoras en relaciona a las otras. Con cada alternativa el modelo de data panel recibe un nombre (efectos fijos, aleatorios, dinámicos etc.).

Se comprueba entonces la forma del error y se escoge mediante la prueba de Hausman si existen efectos fijos o aleatorios. Además, se corrigen los problemas de Heterocedasticidad. El software para realiza estas operaciones es STATA 15.

La eficiencia de estas estimaciones se medirá con el indicador $\mathrm{R}^{2}$, que entre más alto más efectivo, el modelo es mejor en determinar el comportamiento de la variable dependiente.

\section{Resultados}

La Tabla 1 muestra los resultados de las estimaciones de la primera etapa con los coeficientes de cada una de las variables de control (independientes) sobre la probabilidad de acceso a fondos externos. La eficacia del modelo es aceptable pues todas las variables independientes fueron de significancia estadística menor del $5 \%$ y el R2 fue del $25 \%$.

Los coeficientes de las variables continuas muestran cómo cambia la probabilidad de acceder al crédito de la firma si se da un cambio del 1\% en la variable indepen- 
diente de análisis. Por su parte, los betas de las variables categóricas, expresan la magnitud en el cambio en la probabilidad acceder a fondos externos de manera relativa en función de una característica base. Por ejemplo, el porcentaje de los activos de las empresas financiados por medio de fondos internos fueron un $12 \%$ menor en 2017 que en 2010.

TABLa 1.

Relación entre la educación, la experiencia y el salario.

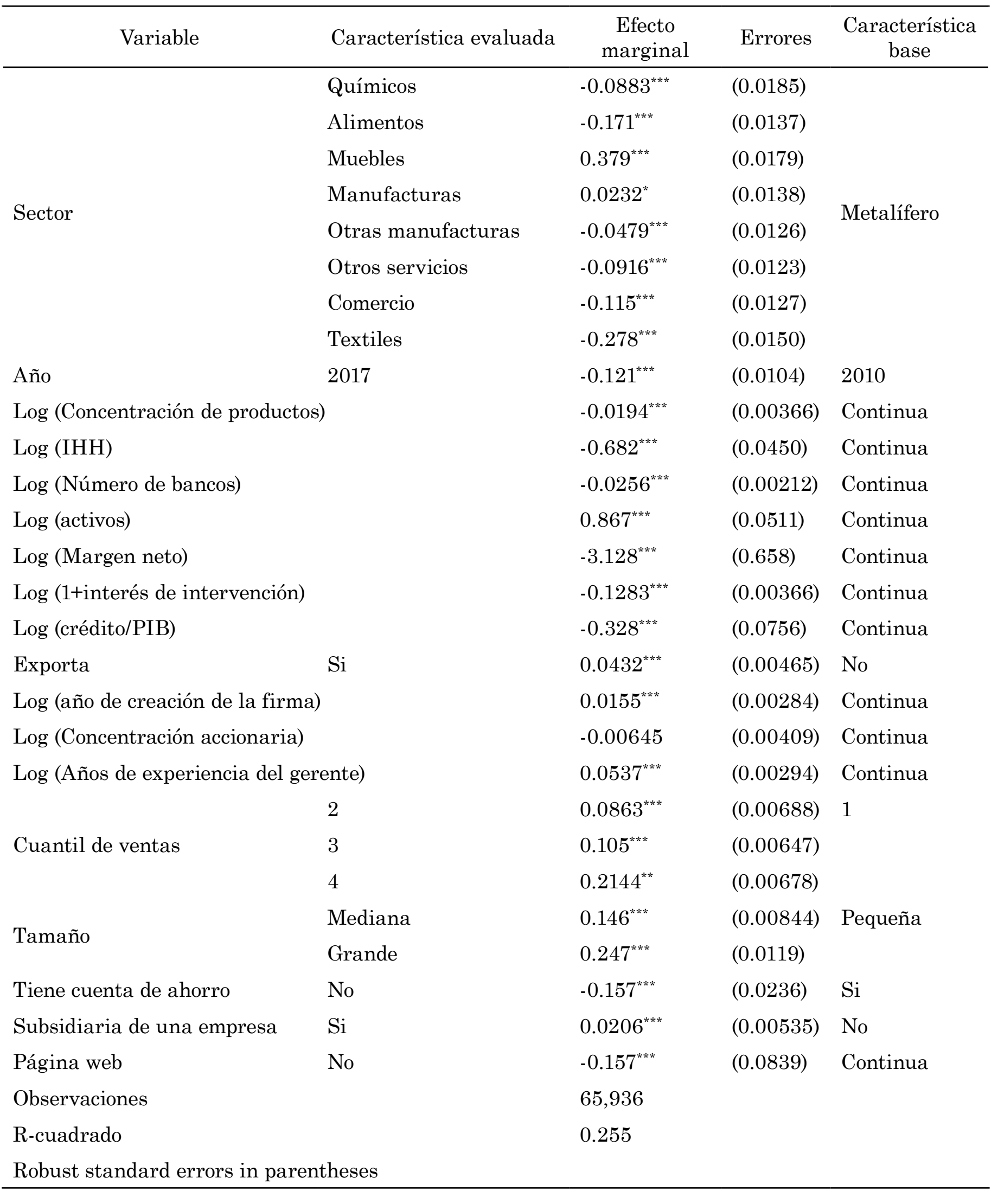

${ }^{* * *} \mathrm{p}<0.01,{ }^{* *} \mathrm{p}<0.05,{ }^{*} \mathrm{p}<0.1$ 
Fuente: Elaboración propia.

Con base en los betas de la Tabla 1 se pasan a revisar cada una de las condiciones señaladas en el diseño metodológico.

H1: El número de empleados y ventas tienen una influencia positiva en la consecución de financiamiento externo. Según las estimaciones, una firma de tamaño grande y mediano tiene entre un $14 \%$ y $24 \%$ más de probabilidad que una pequeña. En cuantiles de ventas, la empresa que se encuentre en el tramo más alto (cuarto cuantil) posee $21 \%$ más de probabilidad que una en el más bajo. Este es el grupo de variable que influye más en el comportamiento de la variable dependiente.

H2: Un incremento del $1 \%$ en los años de funcionamiento de la compañía se ven reflejados en un aumento del $0,14 \%$ y un $0,21 \%$ en la probabilidad del financiamiento externo.

H3: La tenencia de capacidades tecnológicas se asocia positivamente con el acceso al crédito. Si la empresa tiene un sitio web, presenta un $15 \%$ de probabilidad adicional frente a una que no lo tiene. Por su parte, un incremento del $1 \%$ en los años de experiencia del gerente contribuye al 5\%. Entre las características de las organizaciones, una mayor concentración accionaria de la organización favorece el financiamiento externo en un $-0.6 \%$, adicionalmente la tenencia de cuenta bancaria aumenta la probabilidad en un $0.58 \%$.

H4: La tenencia de cuenta de ahorros es un componente positivo de la empresa. Poseerla incrementa la probabilidad de acceso a financiamiento externo en un $15 \%$. Por otro lado, un incremento de la experiencia del gerente, incrementa la probabilidad de acceso al crédito en un $5 \%$.

H5: Las firmas ubicadas en capitales poseen mejores probabilidades de obtener financiamiento externo. En este mismo sentido, las ramas de actividad de los elementos de metal y muebles exponen probabilidades por encima del $37 \%$ frente a las firmas de otros sectores económicos.

H6: Las estimaciones sobre la estructura bancaria apuntan a evidenciar que la mayor concentración financiera afecta negativamente la probabilidad de acceder al crédito. Un incremento del HHI del sector disminuye en $0.682 \%$, el número de bancos en $0.02 \%$ y el incremento del margen neto en $3.12 \%$.

H7: Por su parte, la tasa de interés de intervención del banco central del país de origen disminuye el acceso al crédito en 0.12. La tasa de interés de los fondos federales no fue significativa en el modelo panel de elección discreta.

\section{Conclusiones y Recomendaciones}

El financiamiento externo es necesario para el crecimiento de la actividad empresarial. Sin embargo, en América Latina existen barreras que impiden el desempe- 
ño eficiente de los mercados financieros. Por esta razón, los fondos propios son la fuente de financiamiento más representativa en el balance contable. Solo las empresas grandes pueden hacer uso de los mercados formales mientras las PYMEs hacen frente de colaterales y tipos de interés más elevados. La falta de competencia y la política monetaria puede ser uno de los factores obstaculizadores del acceso al crédito, al restringir la oferta de fondos prestables e imponer altos precios a los servicios financieros. Por esta razón, es deber de los reguladores el brindar las condiciones que permitan la expansión de la frontera de préstamos, llegando a todos tipos de unidades productivas. Asimismo, en función de la globalización de los flujos de capital, la política monetaria exterior puede afectar la capacidad del sistema a proveer fondos prestables.

Por tal motivo, el presente artículo investigó el impacto de la política monetaria de la FED en el acceso al crédito de las empresas latinoamericanas en un panel longitudinal entre 2010 y 2017. Entre los hallazgos, se evidenció que una alta concentración se asocia con la disminución en la probabilidad del acceso al financiamiento de las empresas, yendo en línea con la hipótesis de poder de mercado y la evidencia internacional (Ryan et al., 2014). Lo anterior, incrementa la tendencia de financiarse con fondos propios o informales. Esto tiene implicaciones para los hacedores de política pública, dado su papel de velar por el sostenimiento y estabilidad de las condiciones que promueven un crecimiento del aparato productivo de los países.

Igualmente, la política monetaria interna del Banco Central fue significativa en la regulación del crédito mientras que las tasas de interés externas de la FED no fueron significativas. Estos resultados podrían indicar que los sistemas financieros latinoamericanos no están vinculados de manera directa. Además, se podría inferir que la reducción de los estímulos monetarios de la FED no ha sido significativa. Lo cual no afectado la liquidez mundial.

Para superar las barreras de acceso de las PYMEs es importante incrementar la oferta de productos de crédito a la vez que es necesario crear sistemas de información entre el sector empresarial y las entidades financieras, con el fin de disminuir los costes de transacción que conlleva la asignación de los créditos. Asimismo, se podrían crear programas donde los empresarios puedan profesionalizar la realización de sus estados financieros pues muchos de ellos pueden carecer de los recursos o del conocimiento para realizarlos (Barona. Gómez y Torres, 2006).

Los sistemas nacionales de apoyo destinados a suministrar tasas de interés subsidiadas a los créditos de las PYMEs han sido de los programas utilizados por los gobiernos para subsanar la restricción crediticia. Sin embargo, Held (1999) pone de manifiesto que este mecanismo obstaculiza el desarrollo de los mercados de capital porque no se puede trasmitir un efecto duradero de menores tasas en el mercado. En consecuencia, se distorsionan los esquemas de incentivos de las instituciones financieras para realizar su tarea de valoración del riesgo y puedan solventar el capital dado por sus accionistas.

Una implicación adicional de los hallazgos de este documento, es que si bien no hay consenso sobre una estructura de capital determinada que impulse el crecimiento sostenido de las firmas (De la Torre, Gozzi y Schumukler, 2017), es aceptada la re- 
lación negativa existente entre la ratio de deuda de las empresas y su crecimiento (Wadnipar \& Cruz, 2013). Por lo tanto, un mayor grado de competencia bancaria permitiría mejores condiciones de refinanciamiento para las empresas que lo necesitasen, sin ver afectado su desempeño.

Entre las futuras líneas de estudio están el uso de otros indicadores sobre la competencia, especialmente desde el punto de vista de la eficiencia (indicador de Lerner o de Boone). Lo anterior se podría combinar con la exploración detallada de las realidades propias de cada mercado para proponer soluciones de política efectiva, debiendo explorar los contextos económicos a nivel de regiones.

\section{REFERENCIAS}

Aizenman, J., Binici, M. \& Hutchison, M. M. (2014). The Transmission of Federal Reserve Tapering News to Emerging Financial Markets. International Journal of Central Banking, International Journal of Central Banking, 12(2), 317-356. https://doi.org/10.3386/w19980

Andritzkya, J. R., Bannisterb, G. J. \& Tamirisa, N. T. (2007). The Impact of Macroeconomic Announcements on Emerging Market Bonds. Emerging Markets Review, 8(1), 20-37. https://doi.org/10.1016/j.ememar.2006.05.001

Antoniou, A., Guney, Y. \& Paudyal, K. (2008). The determinants of capital structure: capital market-oriented versus bank-oriented institutions. Journal of Financial and Quantitative Analysis, 43(1), 59-92. Available: https://www.jstor. org/stable/27647340

Arazi, M. C. y Baralla, G. (2012). La situación de las PyMEs en América Latina. [Documento de Trabajo. Año 18 -Edición $\mathrm{N}^{\circ}$ 114]. Córdoba: Inter-American Development Bank (IADB). Recuperado de https://www.ieral.org/images_db/ noticias_archivos/2157-La\%20situaci\%C3\%B3n\%20de\%20las\%20PyMEs\%20 en\%20Am\%C3\%A9rica\%20Latina.pdf

Arellano, M. y Bover, O. (1990). La econometría de datos de panel. Investigaciones económicas, 14(1), 3-45. Recuperado de https://www.cemfi.es/ arellano/arellano-bover-inv-econ-1990.pdf

Arora, V. \& Cerisola, M. (2001). How Does U.S. Monetary Policy Influence Sovereign Spreads in Emerging Markets? IMF Staff Papers, 48(3), 474-498. Available: https://www.imf.org/External/Pubs/FT/staffp/2001/03/pdf/arora.pdf

Avdjiev, S. \& Takáts, E. (2014). El préstamo bancario transfronterizo durante el episodio de taper tantrum: influencia de los fundamentos de las economías emergentes. BIS Quarterly Review, 1-14. Available: https://www.bis.org/publ/ qtrpdf/r_qt1409g_es.htm

BM. (2020). Enterprise Surveys. [Online]. Available: https://espanol.enterprisesurveys.org/es/enterprisesurveys

Barona, B., Gómez, A. y Torres, H. (2006). La financiación de nuevas empresas en Colombia. Las experiencias y opiniones de una muestra de gerentes bancarios. Cuadernos de Administración, 19(32), 45-70. Disponible en https://revistas.javeriana.edu.co/index.php/cuadernos_admon/article/view/4231 
Beck, T., Degryse, H. \& Kneer, C. (2014). Is more finance better? Disentangling intermediation and size effects of financial systems. Journal of Financial Stability, 10(C), 50-64. https://doi.org/10.1016/j.jfs.2013.03.005

Bernanke, B. S. \& Kuttner, K. N. (2005). What Explains the Stock Market's Reaction to Federal Reserve Policy? Journal of Finance, 60(3), 1221-1257. https://doi. org/10.1111/j.1540-6261.2005.00760.x

Bikker, J., Van Rixtel, A. y Kok-Sørensen, C. (2008). Un nuevo indicador de competencia en el sector bancario. Boletín económico-Banco de España, (1), 113-124. Recuperado de https://www.bde.es/f/webbde/SES/Secciones/Publicaciones/InformesBoletinesRevistas/BoletinEconomico/08/Ene/Fich/art5.pdf

Botello, H. (2015). Determinants of access to credit for SMEs: evidence at the level of the firm in Latin America. Apuntes del Cenes, 34(60), 247-276. https://dx.doi. org/10.19053/issn.0120-3053

Boz, G., Menéndez, C., Orgaz, N. y Prior, D. (2015). ¿Influyen las Normas Internacionales de Información Financiera en el riesgo de las acciones? Contaduría y administración, 60(3), 556-577. https://doi.org/10.1016/j.cya.2015.05.010

Burdisso, T. (1997). Estimación de una función de costos para los bancos privados argentinos utilizando datos en panel. [Documento de Trabajo Nro. 3]. Buenos Aires: Banco Central de la República Argentina.

Carbó-Valverde, S., Rodríguez-Fernández, F. \& Udell, G. F. (2009). Bank market power and SME financing constraints. Review of Finance, 13(2), 309-340. https://doi.org/10.1093/rof/rfp003

Cárdenas, M. y Mejía, C. (2007). Informalidad en Colombia: nueva evidencia. Cuadernos de Fedesarrollo, 35, 1-43. Disponible en https://www.repository.fedesarrollo.org.co/handle/11445/805

Chortareas, G., Garza-García, J. y Girardone, C. (2010). Desempeño del sector bancario en algunos países latinoamericanos: Poder de mercado versus eficiencia. [Documento de Investigación, № 20]. México, D.F.: Banco de México.

Claessens, S. \& Laeven, L. (2005). Financial dependence, banking sector competition, and economic growth. Journal of the European Economic Association, 3(1), 179-207. https://doi.org/10.1162/1542476053295322

De Haas, R., y Van Horen, N. (2009). The crisis as a wake-up call. Do banks tighten lending standards during a financial crisis? [Paper MPRA No. 16382]. London: EBRD/DNB. Available at https://mpra.ub.uni-muenchen.de/16382/

De la Torre, M. (2018). Utilización del sector financiero para el lavado de dinero: perspectiva desde la legislación ecuatoriana. Jurídicas CUC, 14(1), 145-166. http://dx.doi.org/10.17981/juridcuc.14.1.2018.7

De la Torre, A., Gozzi, J. C. \& Schumukler, S. L. (2017). Innovative Experiences in Access to Finance: Market-Friendly Roles for the Visible Hand? Latin American Development Forum. Washington, DC: The World Bank. Available: http://hdl. handle.net/10986/27529

De Miguel, A. \& Pindado, J. (2001). Determinants of capital structure: new evidence from Spanish panel data. Journal of Corporate Finance, 7(1), 77-99. https://doi. org/10.1016/S0929-1199(00)00020-1 
Eichengreen, B. y Gupta, P. (2013). The Two Waves of Service Sector Growth. $O x$ ford Economic Papers, 65(1), 96-123. https://doi.org/10.3386/w14968

Fawley, B. \& Neely, C. (2013). Four stories of quantitative easing. Federal Reserve Bank of St. Louis Review, 95(1), 51-88. Available: https://files.stlouisfed.org/ files/htdocs/publications/review/13/01/Fawley.pdf

Gámez, A., Morales, M. C. \& Ramírez, C. (2018). Estado del arte sobre problemáticas financieras de las pymes en Bogotá, Colombia y América Latina. Económicas CUC, 39(2), 77-94. https://doi.org/10.17981/econcuc.39.2.2018.05

García, G. \& Baeza, R. (2016). Las políticas monetarias heterodoxas en el contexto de la Gran Recesión. Un comparativo entre el Sistema de la Reserva Federal y el Banco Central Europeo. Análisis Económico, 31(77), 177-204. Disponible en http://www.analisiseconomico.azc.uam.mx/index.php/rae/article/view/55

Gonzalez, V. M. \& González, F. (2008). Influence of bank concentration and institutions on capital structure: New international evidence. Journal of Corporate Finance, 14(4), 363-375. https://doi.org/10.1016/j.jcorpfin.2008.03.010

Granda, H. (2012). Factores determinantes de acceso y racionamiento del crédito en las MIPYMEs ecuatorianas. [Working paper]. Lima: FLACSO-MIPRO. Disponible en https://repositorio.flacsoandes.edu.ec/handle/10469/9246

Greenwood, J., Sánchez, J. M. \& Wang, C. (2013). Quantifying the impact of financial development on economic development. Review of Economic Dynamics, 16(1), 194-215. https://doi.org/10.1016/j.red.2012.07.003

Held, G. (1999). Políticas de crédito para empresas de menor tamaño con bancos de segundo piso: experiencias recientes en Chile, Colombia y Costa Rica. Santiago de Chile: CEPAL. Disponible en https://www.cepal.org/es/publicaciones/5291politicas-credito-empresas-menor-tamano-bancos-segundo-piso-experiencias

Hofmann, B. \& Bogdanova, B. (2012). Taylor rules and monetary policy: a global "Great Deviation"? BIS Quarterly Review, 37-49. Available: https://www.bis. org/publ/qtrpdf/r_qt1209f.htm

Jiménez, L. F. y Manuelito, S. (2011). América Latina: sistemas financieros y financiamiento de la inversión. Diagnósticos y propuestas. Revista de la CEPAL, (103), 47-75. https://doi.org/10.18356/050898ab-es

Lago, R., López, J. A. \& Saurina, J. (2007). Determinants of access to external finance: evidence from Spanish firms. [Working Paper Series 22]. San Francisco: Federal Reserve Bank of San Francisco. Available: https://www.frbsf.org/ economic-research/publications/working-papers/2007/22/

Love, I. \& Martínez S. (2014). How bank competition affects firms' access to finance. The World Bank Economic Review, 29(3), 413-448. https:/doi.org/10.1093/wber/ lhu003

Martínez, A., Zuleta, L. A., Misas, M. y Jaramillo, L. (2016). La competencia y la eficiencia en la banca colombiana. [Libros de Fedesarrollo No. 015211]. Bogotá, D.C.: Fedesarrollo/Asobancaria. Disponible en https://www.repository.fedesarrollo.org.co/handle/11445/3298 
Maudos, J. (2016). La concentración regional del mercado bancario. Cuadernos de información económica, (251), 49-62. Recuperado de https:/www.funcas.es/wpcontent/uploads/Migracion/Articulos/FUNCAS_CIE/251art07.pdf

Medina, E. (2003). El uso de los modelos de elección discreta para la predicción de crisis cambiarias: el caso latinoamericano. [Tesis doctoral]. Universidad Autónoma de Madrid, Madrid, España. Disponible en http://hdl.handle.net/10486/130857

Miyajima, K., Mohanty, M. y Chan, T. (2015). Emerging market local currency bonds: Diversification and stability. Emerging Markets Review, 22(C), 126-139. https://doi.org/10.1016/j.ememar.2014.09.006

Nguyen, H.-L. Q. (2019). Are credit markets still local? Evidence from bank branch closings. American Economic Journal: Applied Economics, 11(1), 1-32. https:// doi.org/10.1257/app.20170543

Pérez, C. (2004). Técnicas de análisis multivariante de datos. Aplicaciones con SPSS. Madrid: Pearson.

Quicazan, C., Fernández, D. \& Estrada, M. (2012). Credit determinants and their impact on firm's growth in Colombia. Temas de Estabilidad Financiera, (67), 1-25. https://doi.org/10.32468/tef.67

Rai, V. \& Suchanek, L. (2014). The Effect of the Federal Reserve's Tapering Announcements on Emerging Markets. [Staff Working Papers]. Toronto: Bank of Canada. Available: https:/www.bankofcanada.ca/wp-content/uploads/2014/11/ wp2014-50.pdf

Ramírez, J. \& Sarmiento, J. (2020). Nuevas tendencias geopolíticas globales. Económicas CUC, 41(2), 102-114. https://doi.org/10.17981/econcuc.41.2.2020.Org.6

Rice, T. \& Strahan, P. E. (2010). Does credit competition affect small-firm finance? The Journal of Finance, 65(3), 861-889. https://doi.org/10.1111/j.15406261.2010.01555.x

Rivera, J. A. (2007). Estructura financiera y factores determinantes de la estructura de capital de las pymes del sector de confecciones del Valle del Cauca en el periodo 2000-2004. Cuadernos de administración, 20(34), 191-219. Disponible en https://revistas.javeriana.edu.co/index.php/cuadernos_admon/article/ view/4050

Ryan, R. M., O'Toole, C. M. \& McCann, F. (2014). Does bank market power affect SME financing constraints? Journal of Banking \& Finance, 49, 495-505. http://dx.doi.org/10.1016/j.jbankfin.2013.12.024

Urdaneta M. A., Pérez, M. \& Acurero, M. (2018). Enfoque de la política fiscal en Venezuela en el período 2000-2016. Económicas CUC, 39(2), 95-108. https://doi. org/10.17981/econcuc.39.2.2018.06

Vives, X. (2001). Competition in the changing world of banking. Oxford review of economic policy, 17(4), 535-547. https://doi.org/10.1093/oxrep/17.4.535

Wadnipar, S. M. y Cruz, J. S. (2013). Determinación de la estructura de capital de las empresas colombianas. Revista Soluciones de Postgrado, 1(1), 23-44. Disponible en https://revistas.eia.edu.co/index.php/SDP/article/view/2 


\section{BIODATA}

Héctor Alberto Botello Peñaloza es Magister en Ciencias Económicas de la Universidad Nacional de Colombia. Investigador del grupo de planeación territorial (GIDROT) de la Universidad Industrial de Santander (Colombia). Intereses de revisión Desarrollo, Competitividad empresarial y Política monetaria. https://orcid. org/0000-0002-7795-2590

\section{Anexos}

Anexo 1.

Distribución de la encuesta de empresas del Banco Mundial por tamaño y año

\begin{tabular}{llllllllc}
\hline \multirow{2}{*}{ País } & \multicolumn{4}{c}{ Año 2010} & \multicolumn{5}{c}{ Año 2017} \\
\cline { 2 - 8 } & Grandes & Medianas & Pequeñas & Total & Grandes & Medianas & Pequeñas & Total \\
\hline Argentina & 936 & 1,495 & 1,612 & 4,043 & 923 & 1,456 & 1,664 & 4,043 \\
Bolivia & 605 & 594 & 561 & 1,760 & 583 & 605 & 572 & 1,760 \\
Colombia & 1,032 & 1,392 & 1,200 & 3,624 & 1,164 & 1,176 & 1,284 & 3,624 \\
República & 546 & 840 & 630 & 2,016 & 609 & 714 & 693 & 2,016 \\
Dominicana & & & & & & & \\
Ecuador & 242 & 451 & 253 & 946 & 297 & 440 & 209 & 946 \\
El Salvador & 994 & 1,078 & 1,022 & 3,094 & 868 & 1,134 & 1,092 & 3,094 \\
Guatemala & 715 & 611 & 689 & 2,015 & 728 & 494 & 793 & 2,015 \\
Honduras & 216 & 348 & 468 & 1,032 & 156 & 348 & 528 & 1,032 \\
Nicaragua & 273 & 572 & 702 & 1,547 & 299 & 663 & 585 & 1,547 \\
Paraguay & 384 & 468 & 504 & 1,356 & 432 & 456 & 468 & 1,356 \\
Perú & 880 & 1,080 & 1,008 & 2,968 & 1,016 & 1,024 & 928 & 2,968 \\
Uruguay & 407 & 495 & 330 & 1,232 & 385 & 517 & 330 & 1,232 \\
Total & 7,230 & 9,424 & 8,979 & 25,633 & 7,460 & 9,027 & 9,146 & 25,633 \\
\hline
\end{tabular}

Fuente: Elaboración del autor con datos del Enterprise Survey (BM, 2020). 